This document is the unedited Author's version of a Submitted Work that was subsequently accepted for publication in ACS Sensors, copyright $(\subset$ American Chemical Society after peer review. To access the final edited and published work see: https://dx.doi.org/10.1021/acssensors.8b00789. 


\section{Label-free bacteria quantification in blood plasma by a bioprinted microarray based interferometric point-of-care device}

Priyanka Dey ${ }^{1}$, Nuria Fabri-Faja ${ }^{1}$, Olalla Calvo-Lozano ${ }^{1}$, Roland A. Terborg ${ }^{2}$, Alexander Belushkin ${ }^{3}$,

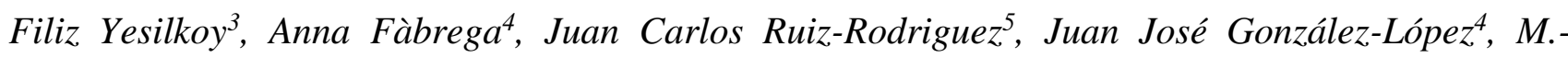
Carmen Estévez ${ }^{1 *}$, Hatice Altug ${ }^{3}$, Valerio Pruneri ${ }^{2,6}$ and Laura M. Lechuga ${ }^{1}$

1) Nanobiosensors and Bioanalytical Applications Group, Catalan Institute of Nanoscience and Nanotechnology (ICN2), CSIC, CIBER-BBN and BIST, Campus UAB, 08193 Bellaterra (Barcelona), Spain.

2) ICFO-Institut de Ciències Fotòniques, The Barcelona Institute of Science and Technology, 08860 Castelldefels (Barcelona), Spain.

3) Institute of Bioengineering, École Polytechnique Fédérale de Lausanne (EPFL), Lausanne 1015, Switzerland.

4) Hospital Universitari Vall d'Hebron. Universitat Autònoma de Barcelona (Barcelona), Spain.

5) Intensive Care Department. Vall d'Hebron University Hospital. Shock, Organ Dysfuction and Resuscitation Research Group. Vall d'Hebron Institute of Research. Barcelona. Spain

6) ICREA—Institució Catalana de Recerca i Estudis Avançats, 08010 Barcelona, Spain.

*Correspondence: MCE: mcarmen.estevez@icn2.cat

\section{$\underline{\text { Abstract }}$}

Existing clinical methods for bacteria detection lack in speed, sensitivity and importantly in Point-ofCare (PoC) applicability. Thus, finding ways to push the sensitivity of clinical PoC biosensing technologies is crucial. Aiming that, we here report a portable PoC device based on Lens-free Interferometric Microscopy (LIM). The device employs high performance nanoplasmonics and custom bioprinted microarrays and is capable of direct label-free bacteria (E. coli) quantification. With only one-step sample handling we offer a sample-to-data turnaround time of 40 minutes. Our technology features detection sensitivity of a single bacterial cell both in buffer and diluted blood plasma and is intrinsically limited by the number of cells present in the detection volume. When employed in a hospital setting, the device has enabled accurate categorization of sepsis patients (infectious SIRS) from control groups (healthy individuals and non-infectious SIRS patients) without false positives/negatives. User-friendly on-site bacterial clinical diagnosis can thus become a reality. 


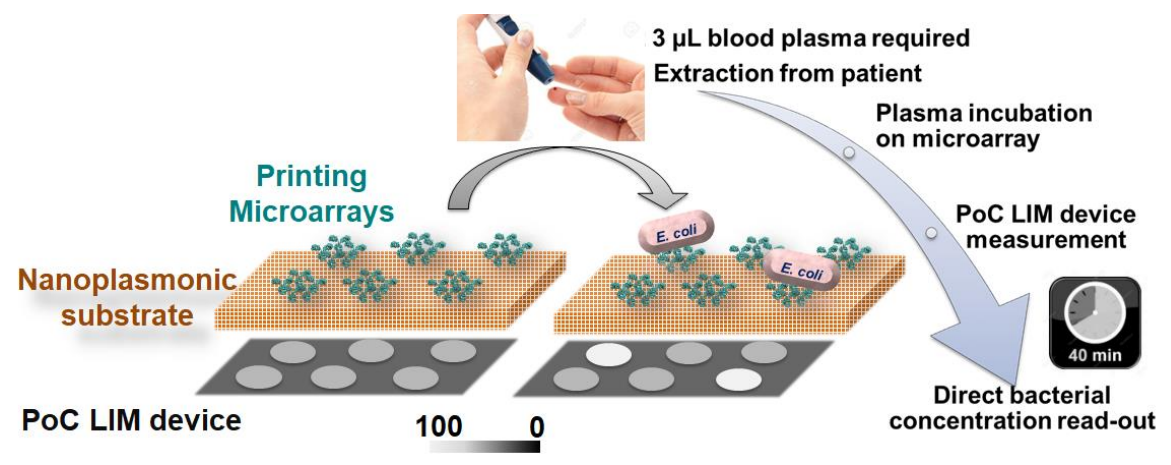

\section{Introduction}

Fast spreading bacterial infections induce acute infections, critical illnesses as meningitis, as well as life-threatening conditions as sepsis. ${ }^{1,2}$ In particular, sepsis with a mortality rate of $\sim 30 \%{ }^{3-7}$ requires fast and accurate diagnosis as the survival chances decreases by $7-8 \%$ for every hour that the infection remains untreated.$^{8-10}$ Rapid, sensitive and quantifiable bacterial detection from patient blood is thus a clinical demand. Blood culture is still the gold standard method used to perform a microbiological diagnosis of sepsis ${ }^{6,11,12}$, but its slow turnaround times delay the delivery of optimal personalized therapy. Furthermore, its low sensitivity results in a positive detection of the pathogen in approximately only $50 \%$ of cases. False-negative results can also be the consequence of presence of antibiotics in blood or the inability of the bacteria to grow under the laboratory culture conditions. ${ }^{13}$ Recently, molecular diagnostic tests requiring a polymerase chain reaction (PCR) amplification step is becoming popular. Although they are labour-intensive, they have improved the sensitivity in addition to reducing the processing time to $\sim 3-8 \mathrm{~h}^{4,8,14}$, but is still not optimal for sepsis treatments. Outdoor or post-operative patients suspected of bacterial infections need rapid diagnosis and categorization $^{15}$ into bacteraemia, sepsis or non-infectious SIRS i.e, Systemic Inflammatory Response Syndrome (a non-infectious process exhibiting similar symptoms to that of sepsis and hence requiring different treatments). Appropriate treatment would thus be possible with sensitive accurate and fast $\left(<1 \mathrm{~h}\right.$, as per hospitals) detection. Bacteria detection with various other technologies like SPR ${ }^{16}$, bimodal waveguide ${ }^{17}, \operatorname{Raman}^{18}$, as well as fluorescence-based smartphone ${ }^{19,20}$ devices have been reported, but most of them have not attempted detection from biofluids like blood or plasma and specifically not addressed sepsis diagnosis. Thus, a rapid sensitive direct and affordable method for bacterial detection with feasibility of hospital on-site testing (i.e., requiring minimal operational expertise) depicted as a clinical point-of-care (PoC) device, is the need of the hour. 
Within the framework of a European project (H2020 RAIS Project, http://www.rais-project.eu/) we have developed an integrated portable and stand-alone instrument based on optical interferometry which when employed with specialized nanoplasmonics can directly detect bacterial cells from patient blood plasma. In order to demonstrate its usability as a PoC device, we customized our assay for specifically targeting Escherichia coli (E. coli). In addition, real patient samples were analysed in a hospital setting using a simple one-step process with sample-to-data turnaround time of 40 minutes. E. coli, a gram-negative bacteria, was selected as the target as it is one of the major causative agents for approximately $30 \%$ of bacterial infections including sepsis. ${ }^{13,21,22}$ Our technology operates with low sample volumes $(10 \mu \mathrm{L})$ and is a quick (40 minutes) one-step quantification method without the need of multiple expensive laboratory instruments, reagents or skilled technicians, thereby offering a user-friendly fast and sensitive clinical PoC device. Our approach paves the way for modern implementable PoC diagnostics in the clinical settings for pathogen detection.

\section{Results and Discussion}

\section{Concept and novelty of the technology}

To attempt bacterial detection in hospital settings, we here present an in-house built portable optical device based on lens-free interferometric microscopy (LIM). Figure 1 shows an overview (left) of the developed PoC device (middle) and its working principle (right) for E. coli detection. The LIM device, recently reported ${ }^{23}$, measures the optical phase shift due to the accumulated mass (contribution of biomolecular height and its surface density) of the designed assay, providing an optical path difference (OPD) value, translating into E. coli quantification in cells/mL. The sensor substrate is based on extraordinary transmission characteristics of the fabricated plasmonic gold nanohole substrate $^{24}$ with holes of $200 \mathrm{~nm}$ diameter and $600 \mathrm{~nm}$ period and an area of $1 \mathrm{~cm}^{2}$ sensor substrate (shown in inset with substrate holder). When the nanohole sensor substrate is inserted into the LIM device along with the sample holder it forms a sandwiched configuration between two polarizers P1 and P2, and savart plates SP1 and SP2, pairs. A collimated light emitting diode (LED) source (660 $\mathrm{nm}$ ) is split by SP1 into two orthogonally polarized beams sheared with respect to each other (shear distance is $50 \mu \mathrm{m}$ ). After passing though the microarray biofunctionalized nanoplasmonic substrate the two sheared beams recombine through SP2 and are further interfered by P2, which is orthogonal to P1. The optical phase shift is thus recorded onto the CMOS image sensor $\left(\sim 25 \mathrm{~mm}^{2}\right)$. The LEDs are chosen to spectrally overlap with the extraordinary transmission peak positions of the gold nanohole substrate in air at $660 \mathrm{~nm}$. The nanohole sensor substrate upon customized microarray based 
biofunctionalization (shown in circular inset) will be capable of specifically capturing E. coli from patient blood plasma. The captured target physically changes the topography of the surface, which is measured with the lens-free interferometric microscope (LIM) PoC device as shown in figure 1 middle. The device incorporates a LED light source and a low-cost CMOS detector in contrast to lasers and CCD cameras, making it cost-effective. Additionally, to minimize operational costs, we utilize a waferscale nanoplasmonic gold nanohole substrate fabrication technology. Finally, cost is also reduced by handling minimal reagents at the testing site as our approach of simple one-step direct label-free detection eliminates usage of secondary antibodies or nanoparticles and thus allows for testing in PoC settings without the need of highly trained laboratory technicians. Furthermore, we attempt to obtain fast $(<1 \mathrm{~h})$ detection from a low blood plasma volume. The device features dimensions of $20 \times 14 \times 23$ $\mathrm{cm}$ and weighs approximately 3-4 kg which provides both compactness and portability and meets the crucial requirements for a clinical PoC device. With such combination of features the technology presents itself as an ideal candidate for PoC medical diagnostics.
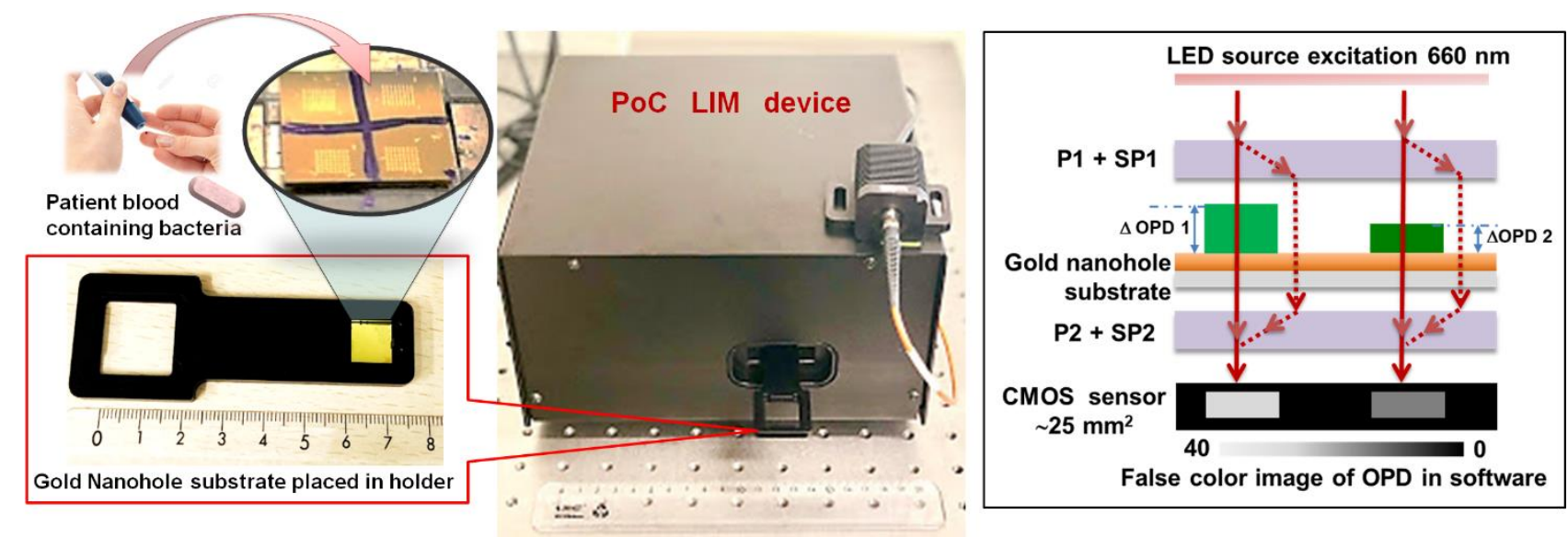

Figure 1. Overview of the PoC LIM technology. Column-wise: (Left) sample from patient for detection, diluted blood plasma sample incubation onto microarray biofunctionalized nanoplasmonic substrate, nanoplasmonic sensor substrate fitted into the PoC sample holder and further inserted into the (middle) LIM PoC device, and (right) the working principle of LIM device.

Bioprinting microarrays as an integral part of the biofunctionalization strategy. The LIM device configuration (depicted in figure 1, right) offers a large field of view of around $25 \mathrm{~mm}^{2}$ with an ultrasensitive axial topographic sensitivity and enables the simultaneous imaging of thousands of discrete small sensing areas ( $\mu \mathrm{m}$ size) with a high lateral resolution. These features emphasize the potential of using bioprinted microarrays as customized sensor substrates rather than uniform surface 
functionalization techniques. Hence, we opted to explore printing biomolecular microarrays via dippen nanolithography (DPN) techniques ${ }^{25}$, which offers high versatility (i.e. by varying the printing ink biomolecular composition with proteins, peptides, DNA or antibodies, etc.) to suit our needs ${ }^{26}$. For our application, specific antibodies with the capability of recognizing surface antigens of the target E. coli were chosen as the capture bio-element. In particular, polyclonal antibodies were selected as they could recognize a variety of different epitopes on a single cell. To maximize the recognition potential of the antibodies, its appropriate orientation is crucial, especially in a label-free microarray based biofunctionalization approach, where area and amount of recognition elements might be a limiting factor to reach desirable sensitivity. Therefore, instead of bioprinting antibodies directly onto the substrate, it was essential to employ another biomolecule which could steer the antibody to a preferable orientation for optimal target detection. We achieved this by employing protein $\mathrm{G}$, which in its native form has high affinity for binding to both the Fc and Fab fragments of an antibody. We especially used a recombinant protein $\mathrm{G}$ which lacks binding sites to Fab regions, thus ensuring only the capture through the $\mathrm{Fc}$ region and maximizing the desired tail-on orientation ${ }^{27}$. We thus bioprinted protein $\mathrm{G}$ via the high-resolution patterning technique of dip pen nanolithography as shown in figure $2 \mathrm{~A}$. A series of experiments (details in Supporting Information section 1, figure S1) were performed in order to optimize printing conditions and were chosen to obtain maximum OPD signal. With the optimised conditions, the printing of $8 \times 8$ array with $250 \mu \mathrm{m}$ of spacing between individual micro-spots was carried out at a protein $\mathrm{G}$ concentration of $500 \mu \mathrm{g} / \mathrm{mL}$ with $5 \%$ glycerol in PBS with a contact time of 3 s per micro-spot in a controlled humidity chamber (75-80\%) resulting in an individual micro-spot of size $50 \times 55 \mu \mathrm{m}$. Four such micro-arrays were printed onto a $1 \mathrm{~cm}^{2}$ nanoplasmonic sensor substrate, as shown in figure $2 \mathrm{~B}$, which was followed by $2 \mathrm{~h}$ incubation at room temperature and rinsing and drying steps to anchor the protein $\mathrm{G}$ molecules to the gold substrate, as depicted in figure 2C. The SEM images of the bioprinted microarray are shown in figure $2 \mathrm{D}(\mathrm{a})$. Figure $2 \mathrm{D}(\mathrm{b})$ shows the SEM image of one micro-spot printed with optimized conditions, whereas, figure $2 \mathrm{D}(\mathrm{c})$ shows the underlying gold nanohole substrate. 


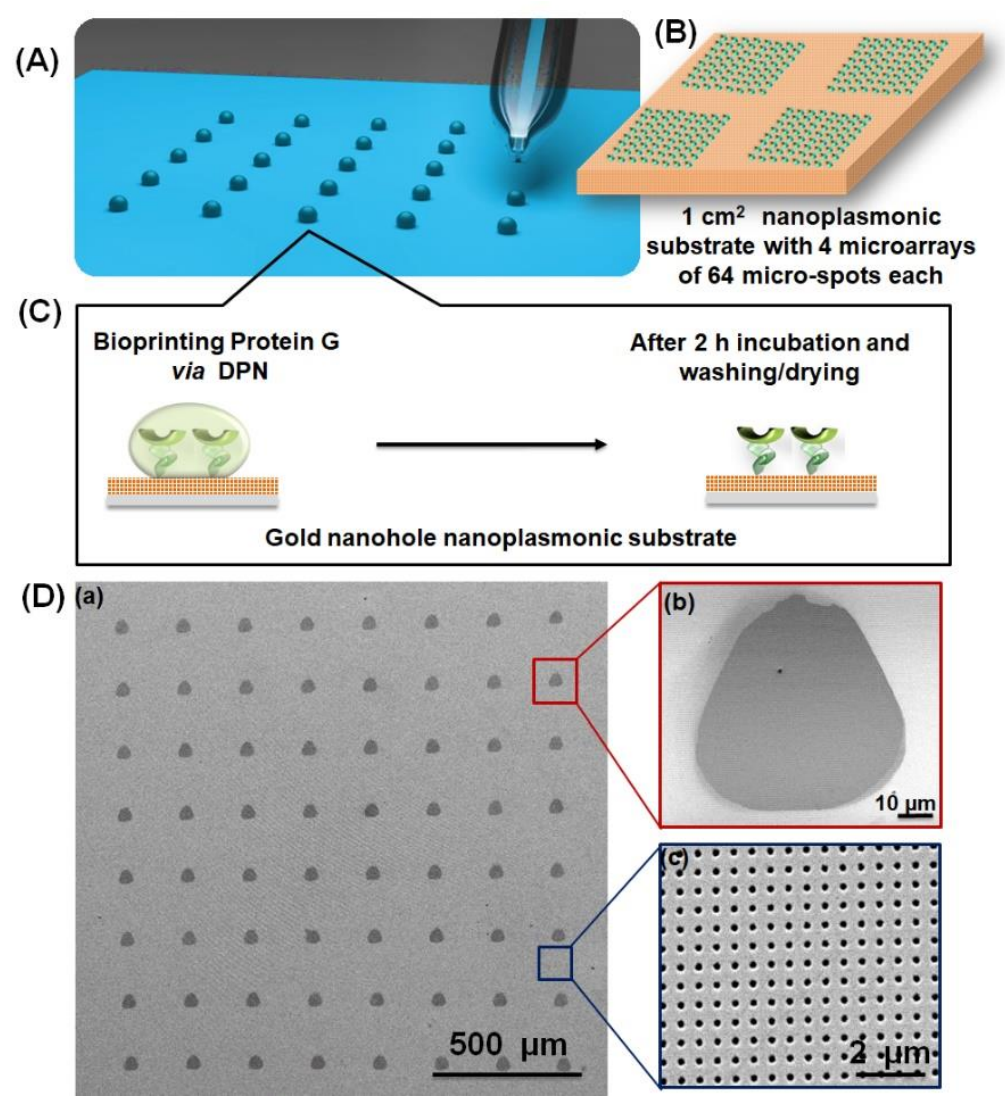

Figure 2. Bioprinting microarrays. (A) Cartoon depiction of bioprinting with dip pen nanolithography (DPN) tip; (B) Cartoon depiction of bioprinted nanoplasmonic substrate of size $1 \mathrm{~cm}^{2}$ which has 4 microarrays, each printed with $8 \times 8$ array i.e., 64 micro-spots. (C) Scheme representation of bioprinting via DPN; (D) SEM images of (a) the bioprinted microarrays of protein $\mathrm{G}$ in $8 \times 8$ array with $250 \mu \mathrm{m}$ of spacing (scale bar $=500 \mu \mathrm{m}$ ), (b) one formed micro-spot of dimensions $\mathrm{L} \times \mathrm{W}$ of $50 \times 55$ $\mu \mathrm{m}$ (scale bar $=15 \mu \mathrm{m}$ ) and (c) underlying gold nanohole array with $200 \mathrm{~nm}$ diameter and $600 \mathrm{~nm}$ period (scale bar $=2 \mu \mathrm{m})$.

\section{Design and validation of the assay}

Bioprinting protein $\mathrm{G}$ at $500 \mu \mathrm{g} / \mathrm{mL}$ directly onto cleaned and hydrophilized gold nanohole surfaces constituted the initial step. The protein $\mathrm{G}$ biomolecules were then left to physically adsorb onto the gold surface at room temperature (RT) for $2 \mathrm{~h}$ which was sufficient for binding via a contribution of electrostatic forces and the amine groups of the protein. A thorough rinse was done at each stage to ensure removal of excess of unanchored biomolecules. Further treatments include blocking non-specific adsorptions onto the non-printed gold surface, as well as anchoring the antibodies to spotted protein G, as schematically depicted in figure 3A. Blocking of the non-occupied gold surface was necessary for minimizing non-specific attachment of blood plasma proteins or other components when analyzing patient blood samples. We employed bovine serum albumin (BSA) as a 
blocking agent which anchors to the gold via electrostatic interactions. Thiolated blocking agents having strong affinity for gold surfaces were strategically avoided to minimize displacement of the protein $\mathrm{G}$ biomolecules. The blocking step also aided in higher loading of antibodies onto the protein G (spotted area), noted as an increase of the OPD signal (see figure S1 in the Supporting Information). This is apprehended to be a combined effect of the inherent higher affinity of antibody to protein $G$ (spotted area) and steric repulsion between antibodies and BSA molecules (unspotted area). A $0.5 \%$ (w/v) of BSA in PBS buffer was used which correlated with employing an excess of a theoretical monolayer of BSA required to cover the unoccupied non-spotted area of $1 \mathrm{~cm}^{2}$ of the gold nanohole substrate. This was followed by incubating the substrate with E. coli specific polyclonal antibodies at a concentration of $500 \mu \mathrm{g} / \mathrm{mL}$. Once the array was rinsed and dried, it was then ready to be incubated with $E$. coli for quantification. Bacterial incubation for 30 minutes in a static mode was employed, as bacterial quantification in hospitals for categorizing infections on-site demands short sample-to-results time. Figure 3B shows the interferometric images with false color scale of the different stages of the biofunctionalization strategy after performing the measurement in air (dry conditions) and figure 3C depicts their respective OPD values. The arrays generated after bioprinting the protein $G$ appear visible, observing OPD values around 30, confirming its binding to the substrate. Both the biomolecule height and its surface density i.e., accumulated mass, is reflected in the OPD signal ${ }^{23,24}$. The blocking step results in OPD signal decrease as the BSA anchors onto the gold surface effectively reducing the

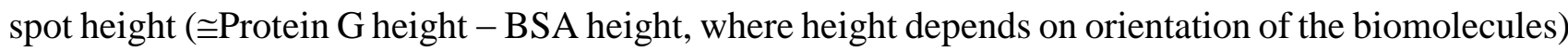
and finally increases significantly $(\sim 75)$ due to antibody attachment (both increase in height and surface density) onto the protein G micro-spots. The bioprinting design exhibits high reproducibility, evident from figure S2 in supporting information, in intra-array, inter-array (microarrays within a $1 \mathrm{~cm}^{2}$ substrate) and inter-substrate (microarrays printed on different substrates). Experiments, as explained in the supporting information figure S3, suggests a high bacterial detection sensitivity with an antibody concentration of $500 \mu \mathrm{g} / \mathrm{mL}$ (as compared to $250 \mu \mathrm{g} / \mathrm{mL}$ ) and larger spot size of $55 \mu \mathrm{m}$ (in contrast to $25 \mu \mathrm{m}$ ) and hence a concentration of $500 \mu \mathrm{g} / \mathrm{mL}$ of the antibody onto the protein $\mathrm{G}$ micro-spots of 55 $\mu \mathrm{m}$ was finally selected as the optimal one. 
(A)

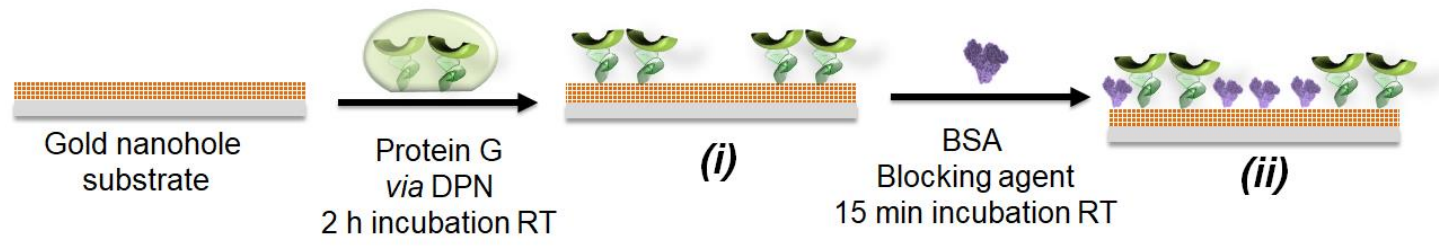

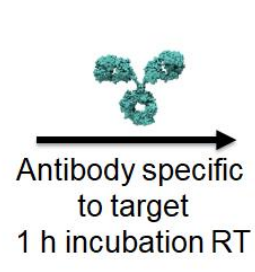

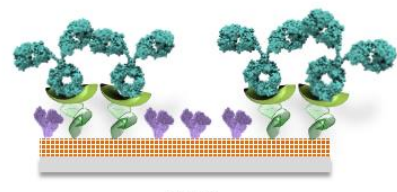

(iii)

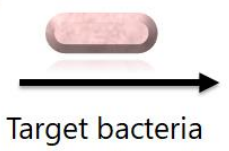

Escherichia coli

30 min incubation RT

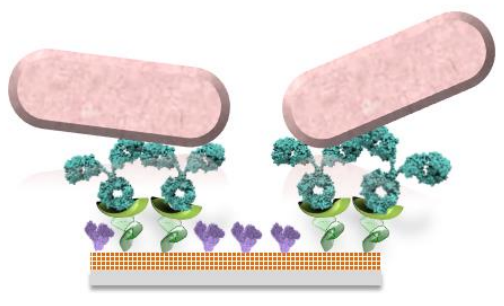

(B)
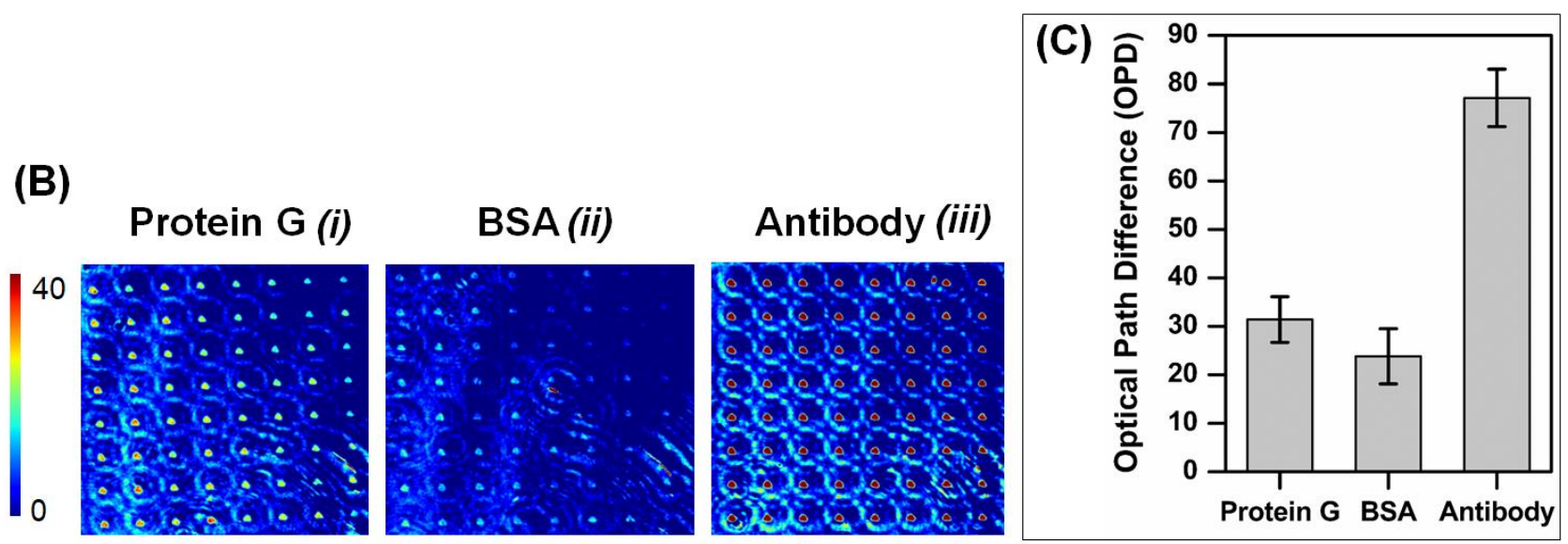

Figure 3. Development of the assay. (A). Biofunctionalization strategy (scheme not to scale), (B) Representative interferometric images for protein $G$ spotting, BSA blocking and antibody immobilization, respectively, with the false colour image indicative of the OPD intensity. (C) Mean OPD signal ( $\pm \mathrm{SD})$ obtained for inter-array ( $8 \times 8$ i.e, 64 micro-spots).

Considering that a total of 181 different serotypes of $E$. coli have been classified and different serotypes of $E$. coli have been associated with bacterial infections, especially with sepsis, ${ }^{21}$ polyclonal antibodies specific for most serotypes of $E$. coli were preferred. Three different polyclonal antibodies pAb (ab31499 rabbit pAb, rPAB80 rabbit pAb and mPAB1:13.5 mouse pAb) were evaluated for performance (see figure S4 in the Supporting Information). The antibody immobilization ( $\mathrm{OPD}_{\text {antibody }}$ - OPD ${ }_{\text {BSA }}$ ) was similar for rPAb80 and ab31499 while resulting in lower OPD signals for mPAb1:13.5. Furthermore, we observed a significantly higher E. coli detection sensitivity with ab31499 than the others. Thus, ab31499 was selected for the assay development for E. coli detection.

Being a whole-cell quantification method, it was crucial to evaluate two relevant factors. Firstly, as we aimed at detecting discreet entities of bacteria, the volume of the sample was highly critical 
especially at low concentrations (i.e., a concentration of 1000 cells $/ \mathrm{mL}$ results in the presence of 10 cells when the sample volume is as low as $10 \mu \mathrm{L}$ and 500 cells for a volume of $0.5 \mathrm{~mL}$ ). Secondly, determining the OPD signal enhancement resulting from averaging OPD signal of an array with several spots (i.e. $8 \times 8=64$ micro-spots) could introduce a high variability, as at lower range of concentrations the bacteria may not be uniformly distributed on all the micro-spots (see figure 4A for a representative image, where the OPD signal increases (red) in certain spots as compared to others (greenish), depicting high spot-to-spot variability). Therefore, the most efficient approach to account for this variability is to calculate a total OPD enhancement $\mathrm{OPD}_{\text {total }}$ (as shown below) which is the summation of the $\triangle \mathrm{OPD}$ of each micro-spot $i$ (considering only those micro-spots with $\Delta \mathrm{OPD}_{i}>0$, due to $E$. coli capture) of the entire microarray with $n$ micro-spots i.e., $i=1$ to $n$ (here, $n=64$ ).

$$
\mathrm{OPD}_{\text {total }}=\sum_{1}^{n} \Delta O P D_{i} \quad \text { where } \triangle O P D_{i}=O P D_{\text {sample }_{i}}-O P D_{\text {antibody }_{i}}
$$

Introducing this calculation approach a calibration curve in PBS was obtained as shown in figure 4B. The samples were analyzed in triplicates and a mean $\mathrm{OPD}_{\text {total }}$ value was obtained with an interarray standard deviation. Importantly, we were also able to estimate the bacterial load over several log orders, $10^{2}-10^{6}$ cells $/ \mathrm{mL}$, with merely $10 \mu \mathrm{L}$ sample volumes. When employing $10 \mu \mathrm{L}$ volume, a lower limit of detection (LOD) of 100 cells/mL (as shown in fig 4B) was observed, while using a volume of $150 \mu \mathrm{L}$ a LOD of 8 cells/mL (as shown in the inset of figure 4B, marked in blue) was achieved, where both were equivalent to 1 cell in the employed detection volume. As the system was limited by the maximum static droplet size of $\sim 150 \mu \mathrm{L}$ volume (for target incubation) onto the $1 \mathrm{~cm}^{2}$ size nanoplasmonic substrate without overflowing, the experimental LOD attainable in PBS was 8 cells/mL. Therefore, we could successfully detect 1 bacterial cell present in the employed detection volume experimentally. The specificity and selectivity of the assay was also evaluated with control experiments summarized in figure 4C. A control bacteria (Bacillus cereus) was tested on the E. coli specific antibody array. For a similar concentration of bacteria (E. coli and B. cereus), the signal $\left(\mathrm{OPD}_{\text {total }}\right)$ was much lower for B. cereus $\left(\mathrm{OPD}_{\text {total }}=18.9\right)$ compared to $E$. coli $\left(\mathrm{OPD}_{\text {total }}=91.1\right)($ pink and red curves in figure 4D). ELISA experiments confirmed this signal came from the slight crossreactivity of the antibody for this bacterium. As B. cereus is not a sepsis-causing primary bacterium, this slight signal should not be a major problem. Moreover, other bacteria tested with an ELISA, such as $P$. aeruginosa, which is indeed more relevant in sepsis, showed a much lower cross-reactivity with these specific antibodies, even at higher bacteria concentration (data not shown). Additional tests confirmed the lack of non-specific binding of $E$. coli, when (i) no antibody is present or (ii) a different 
control antibody is used (no specificity towards E. coli) as reflected in the black and blue curves respectively. The accuracy of the developed assay was evaluated by analyzing several blind spiked samples and correlating it with the calibration curve (see figure 4D). We obtained a linear relationship between the real and the calculated concentrations of the blind samples with a high correlation coefficient. As compared to the reports for bacterial detection with biosensors ${ }^{17,28-32}$, the experimental detection limit of 8 cells/mL in PBS in just 40 minutes with high specificity in a label-free optical biosensor technology is a giant step ahead in this domain. As our main goal was to provide PoC medical diagnostics, we investigated its applicability for the evaluation of patient samples in a hospital setting.

(A)
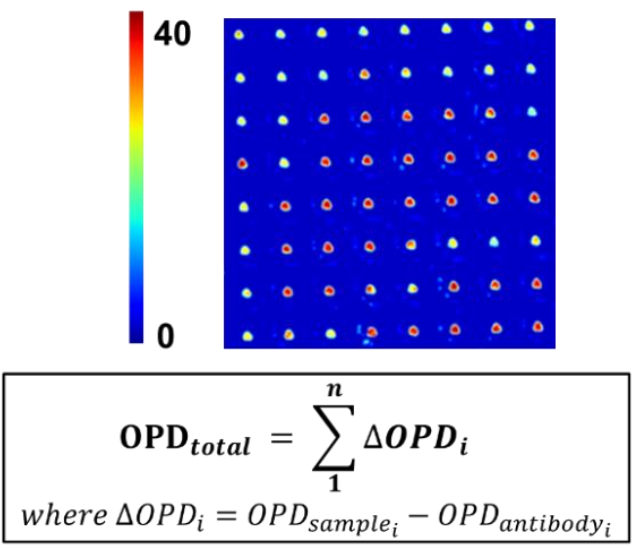

(C)

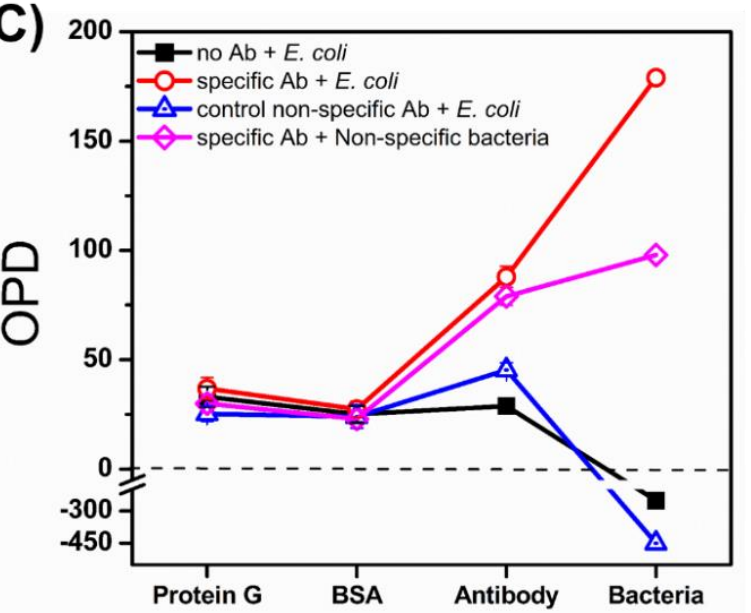

(B)

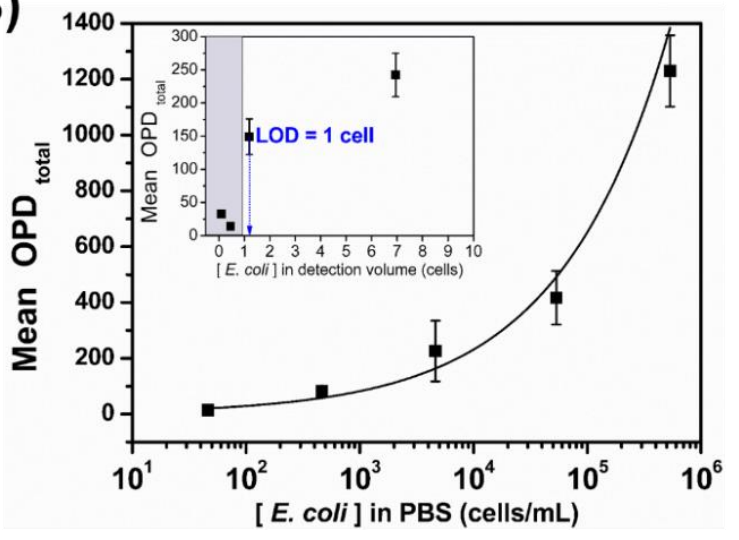

(D)

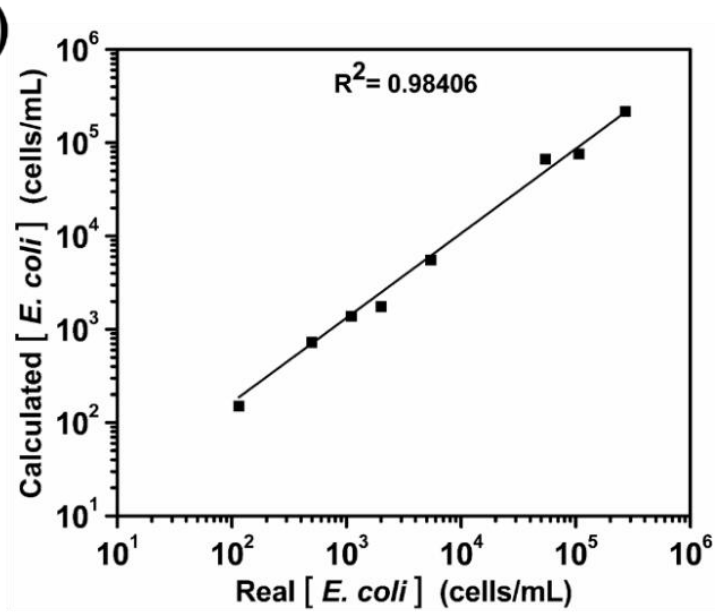

Figure 4. Detection sensitivity and specificity of the assay. (A) Interferometric image after E. coli incubation and the equation used to calculate the $\mathrm{OPD}_{\text {total, }}(\mathrm{B})$ Calibration curve of E. coli in PBS without pre-concentration (i.e., without bacteria enrichment) in a wide dynamic range of 10-10 cells/mL. Inset: Plot indicating the experimental LOD in terms of cells in a constant detection volume of $150 \mu \mathrm{L},(\mathrm{C})$ Specificity tests for the designed and optimized E. coli assay. The graph shows the OPD values obtained in the successive steps. For the biofunctionalization steps, the OPD signal is obtained as the mean OPD signal $( \pm \mathrm{SD})$ for an array $(8 \times 8$ i.e, 64 spots). For bacteria detection, $\mathrm{OPD}_{\text {bacteria }}=\mathrm{OPD}_{\text {antibody }}+\mathrm{OPD}_{\text {total. }}$ Black sequence $:$ no antibody immobilized, E.coli detection (463 
cells/mL in PBS); red sequence: specific antibody immobilized, E. coli detection (463 cells $/ \mathrm{mL}$ in PBS); blue sequence: non-specific control antibody immobilized, E. coli detection ( $463 \mathrm{cells} / \mathrm{mL}$ in PBS); pink sequence: specific antibody immobilized, control non-specific bacteria (B. cereus) detection (590 cells/mL in PBS), and (D) Correlation plot of real and calculated concentrations of blind spiked bacterial samples in PBS.

\section{Validation of the designed assay in blood plasma}

In order to proceed for clinical testing, we firstly evaluated the effect of blood plasma on the developed microarray assay. Although undiluted plasma would be the ideal candidate for any clinical or point-of-care diagnostics, upon its evaluation, we observed that plasma components like proteins anchors to the antibodies and/or the microarray surface and hence contribute significantly to the OPD $_{\text {total }}$ values, which in turn could hinder bacterial detection. Diluting plasma can help minimize this effect. In particular a good trade-off was obtained at 25\% plasma (1:3 v/v diluted with PBS), as shown in figure 5A. Comparing $25 \%$ and 50\% diluted plasma, a lower background, as well as a higher OPD total for $E$. coli quantification was observed for $25 \%$ plasma (white column figure $5 \mathrm{~A}$ ), which could aid in obtaining better assay sensitivity and bacteria detectability. Adding the surfactant tween 20 at $0.1 \%$ (w/v) in PBS (referred to as PBST), we observed even higher OPD total signals as compared to PBS (see figure 5B), further improving the sensitivity of the assay. The diluted blood plasma with the addition of the non-ionic surfactant tween provide a much-needed balance for warding off unnecessary plasma proteins from anchoring to the array and bacteria surface, thus improving antibody-bacteria recognition, resulting in increasing the $\mathrm{OPD}_{\text {total }}$ signals. Serial dilutions of E. coli were then spiked into blood plasma, followed by 1:3 dilution with PBST and analyzed. A calibration curve was obtained with spiked samples in $25 \%$ plasma-PBST with inter-array triplicates. The respective mean OPD total has been plotted in figure 5C. It is worth noticing that even in diluted plasma, a wide range of E. coli concentrations of $10^{2}-10^{5}$ cells $/ \mathrm{mL}$ were detectable. This correlates to a detection range of $1-1000$ cells in $10 \mu \mathrm{L}$ diluted plasma (which employs $<3 \mu \mathrm{L}$ of undiluted blood plasma). More importantly, our assay demonstrates detection sensitivity of 1 bacterial cell in diluted plasma (25\% plasma-PBST) corresponding to 4 bacterial cells in undiluted plasma (100\% plasma) conditions. 

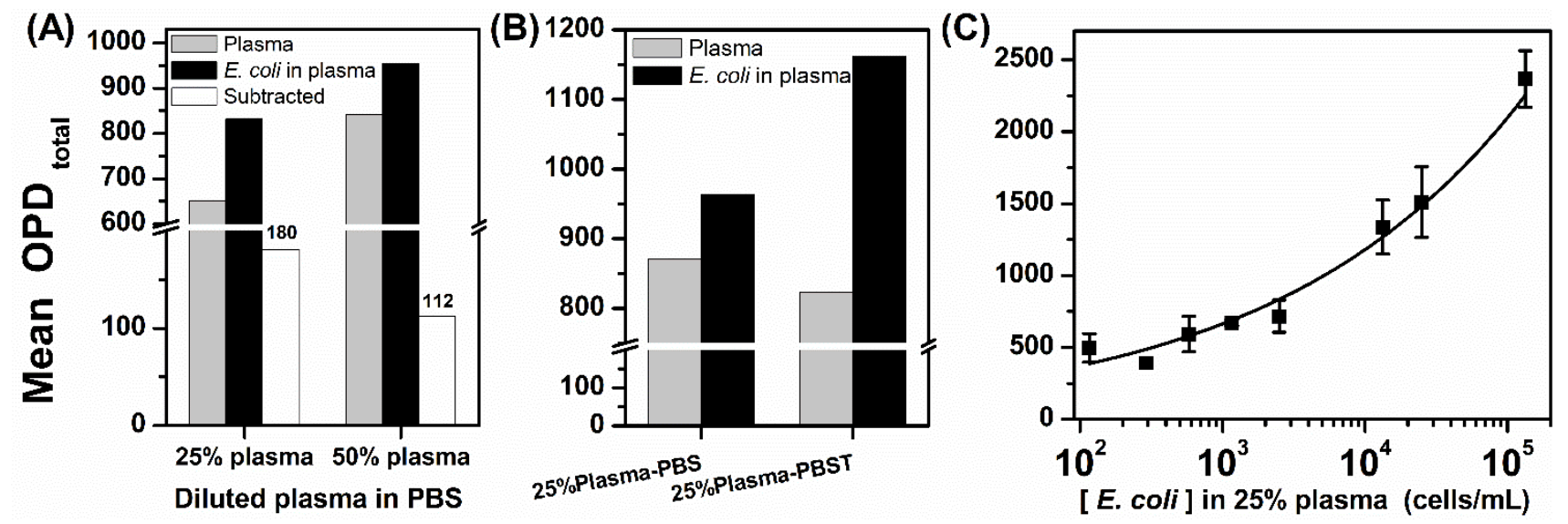

Figure 5. Validation of assay in plasma. (A) Effect of plasma dilution on the nonspecific binding and E. coli detection; (B) Effect of the buffer (PBS and PBST) used for diluting the plasma on the nonspecific binding and E. coli detection, and (C) Calibration curve in $25 \%$ (v/v) diluted plasma in PBST. All Y-axis represent mean $\mathrm{OPD}_{\text {total }}$ values.

\section{Clinical testing}

We evaluated the clinical utility of the biosensor platform using patient blood plasma samples from a hospital. Samples were recovered from the Sepsis Bank located at Vall d'Hebron University Hospital (Barcelona, Spain). Three different category samples, previously collected and stored at the Hospital Biobank facilities, were used: sepsis and control groups (non-infectious SIRS and healthy donors). Blood culture analysis (1-2 days) and pathogen identification by means of molecular diagnosis with IRIDICA platform (Abbott Molecular, Des Plaines, IL) ${ }^{33,34}$ (>8 h) were previously carried out at the Clinical Microbiology Laboratory at Vall d'Hebron University Hospital. The sample data is summarized in Table S1 in the Supporting information. The respective patient EDTA plasma samples were diluted with PBST (1:3 to obtain a $25 \%$ plasma) and incubated for 30 minutes onto the E. coli specific antibody microarray sensor substrates. Following a rinsing step with PBS and milli-Q water and drying with nitrogen, the OPD measurements were carried out with the LIM PoC device in the hospital. Each sample was analyzed in duplicate. $O P D_{\text {total }}$ was calculated and correlated to the calibration curve (figure 5C) providing a quantification of E. coli (cells $/ \mathrm{mL}$ ) in $25 \%$ diluted plasma and thereafter a concentration in undiluted $100 \%$ plasma sample was calculated by quadrupling the above values. The mean and standard deviation data (between the duplicates) has been plotted in figure 6. The step-wise calculations have been built-into an in-house developed software requiring only few minutes. When employing $10 \mu \mathrm{L}$ volume of diluted $25 \%$ plasma, anything below 1 bacterial cell

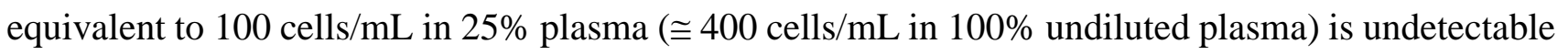


and hence quantification obtained below 400 cells/mL (LOD as marked in figure 6) was considered to be the sole effect of the blood plasma components anchoring to the microarray. As shown in the figure 6, all the healthy controls (section 6a) and non-infectious (no presence of bacteria) SIRS patients (section 6b) provided a concentration below the LOD, whereas a concentration above the LOD was obtained from patients diagnosed with sepsis positive to E. coli (section 6c). The results were corroborated with microbiological culture and/or IRIDICA results. This is a demonstration that the developed technology shows excellent capabilities for discriminating sepsis patients (when E. coli was the causative agent) from healthy controls and, more importantly, from non-infectious inflammatory conditions showing an extremely high sensitivity and specificity, and thus has enormous potential for use at clinical settings close to the patients. Moreover, this could also aid in implementing the appropriate treatment within only an hour after the patient blood is analyzed, thereby boosting practical applicability to patient care, as opposed to approximately 3-8 hours for molecular diagnostics ${ }^{33,34}$ and 1-2 days for blood culture tests where both require trained personnel and expensive laboratory set-ups for operation.

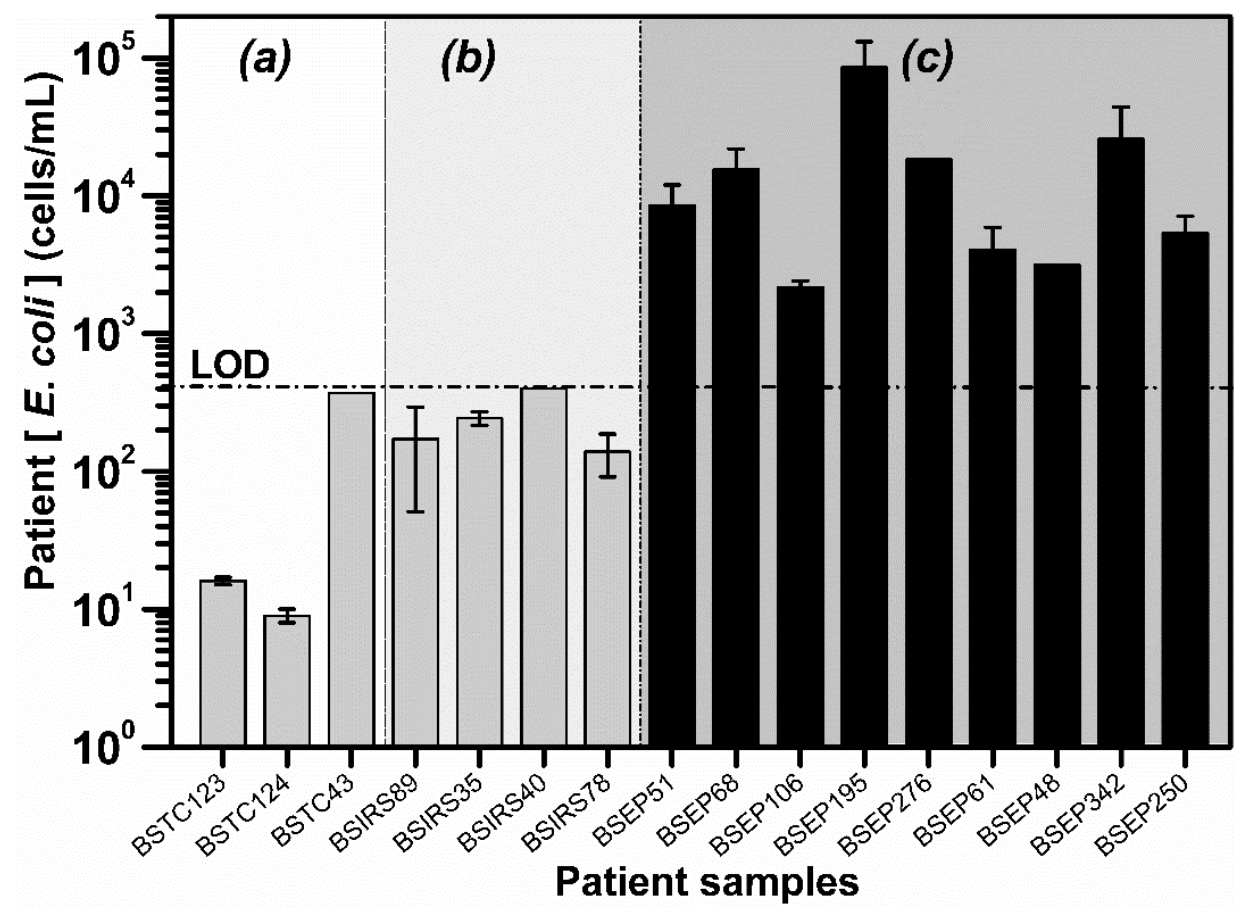

Figure 6. Clinical evaluation of the diagnostic assay with real patient samples. Measurements were done in situ at the hospital settings (a) healthy controls, (b) SIRS patients serving as controls for noninfectious disease and (c) sepsis patients. The indicated concentrations refer to the concentrations in undiluted $100 \%$ plasma. Horizontal dotted line at 400 cells $/ \mathrm{mL}$ indicates the LOD of the assay (in undiluted $100 \%$ plasma). Biobank codes for patient samples have been depicted in X-axis (hospital sample names were anonymised to protect personally identifiable data). 
In regard to the absolute quantification of E. coli, two major factors need to be considered for the interpretation of the values obtained. Firstly, our system being a label-free optical technique suffers from the limitation of variability of the optical properties of the patient plasma itself, which neither can be overlooked nor can be completely accounted for as it inherently varies from patient-to-patient. Although this gives rise to the high signals obtained from control group (healthy and SIRS patients (fig $6 a+b$ ), we here have proven that we can still distinguish between control patients and sepsis patients (multiple patient plasma studied in both cases), justifying the robustness of our PoC technology. Additionally, it is important to note that the detection of the assay is based on the use of specific antibodies targeting bacterial surface antigens (i.e, the antibody used is reactive with all $\mathrm{O}$ and $\mathrm{K}$ antigenic serotypes). According to the commercial source, the immunogen used to produce the antibodies consisted of a mixture of intact and lysed (denatured) bacteria. Therefore, it is likely that the antibody is able to detect both viable bacteria (which can grow in the appropriate media, such as in blood culture) and non-viable bacteria (dead, lysed etc. which remain undetected in blood culture). In fact, our group has previously reported ${ }^{17}$ that the employed polyclonal antibody ab31499 shows a $6 \%$ better sensitivity for lysed bacteria than whole bacteria. We have further verified this by ELISA where a bacteria sample was measured before and after a heating-cooling cycle, which produced lysed bacteria. Despite the decrease in the viable bacteria determined after colony counting, the signal was significantly higher, confirming the employed antibody ab31499 could recognize both viable and lysed bacterial cells, with even higher affinity (i.e. higher signal) when lysed cells are present (data not shown).

Hence, our quantification outcome cannot be strictly correlated with blood culture quantifications. On contrary, molecular tests developed for bacterial identification are carried out in a culture-independent manner. For nucleic acid methods such as PCR results are reported as genome copies (GC) a value that integrates quantification of both viable and non-viable bacterial cells, therefore acknowledging higher bacterial load of $10^{3}-10^{4} \mathrm{GC} / \mathrm{mL}^{31,33,35}$. Overall, the successful and accurate bacterial infection categorization provided by the developed assay with the novel PoC device, obtained within minutes and without the need of highly qualified personnel in a simple one-step analysis (i.e. no secondary antibody or nanoparticle enhancement required), makes it an ideal candidate for clinical PoC diagnostics.

\section{Conclusions}


We have presented a novel PoC device for fast and sensitive E. coli quantification requiring minimal blood plasma volume. This has been achieved by a unique combination of customized microarrays bioprinted onto high performance gold nanohole substrates combined with an in-house built sophisticated and portable LIM device. The tailored bioprinted microarrays featuring antibodytargeted capture of $E$. coli provides detection in a wide dynamic range of concentrations of approximately 5 orders of magnitude in both buffer media and untreated diluted plasma. A high sensitivity of 1 bacterial cell has been achieved both in buffer and diluted plasma conditions and is only fundamentally limited by the number of cells present in the detection volume. The assay involves a direct label-free quantification of $E$. coli without sample pre-processing (like PCR) and/or use of secondary antibodies or labels, providing quantification in a simple one-step process, a crucial requirement for PoC applications. This by itself has potential for direct translation for bacterial load detection in wastewater. Moreover, a further optimized assay was employed for bacterial detection in diluted human plasma, capable of accurately distinguishing samples of bacterial sepsis patients from control group (healthy individuals and SIRS patients). The test can thus be carried out by non-expert personnel at the bed-side of patients, requiring a low blood plasma volume of $3 \mu \mathrm{L}$ with sample-to-data turnaround time of 40 minutes. This will thus have a strong impact in guiding quick medical decisions across various clinical scenarios. Our work including the clinical validation demonstrates the enormous potential of such developed novel optical $\mathrm{PoC}$ device for future clinical applications just by specifically designing customized bioprinted microarrays for specific disease biomarkers.

\section{Methods}

Microarray printing. The gold nanohole substrate was thoroughly cleaned by immersing it for $10 \mathrm{~s}$ in piranha solution, followed by rinsing in milli-Q water for $5 \mathrm{~min}$ to remove traces of acid. This was followed by a treatment in UV chamber for $20 \mathrm{~min}$ and submerging in milli-Q water for a further 30 minutes to impart hydrophilicity to the substrate. All the spotting experiments done for generation of microarray based functionalized sensor substrates have been performed by the ICTS "NANBIOSIS" more specifically by the Unit of Biodeposition and Biodetection (U4) of CIBER in Bioengineering, Biomaterials \& Nanomedicine (CIBER-BBN) at the Catalan Institute of Nanoscience and Nanotechnology (ICN2, Barcelona, Spain). A dip-pen nanolithography-based spotter (BioForce Nanosciences, Utah, US) was used. Specific quill pin tips compatible with the instrument were exposed to oxygen plasma treatment for 2 minutes at $45 \%$ gas flow to reduce hydrophobicity and allow the 
flow of bio-ink for printing. A $4 \mu \mathrm{L}$ of $500 \mu \mathrm{g} / \mathrm{mL}$ of protein $\mathrm{G}$ in PBS buffer was prepared with a net $5 \%(\mathrm{w} / \mathrm{v}$ ) of glycerol (a viscosity regulating buffer) acting as the bio-ink and $0.5 \mu \mathrm{L}$ of this solution was pipetted onto the inlet reservoir of the tip, such that by capillary force the ink moves to the tip outlet and can be used for bioprinting. The microarray bioprinting was performed at $21^{\circ} \mathrm{C}$ and $75-80$ $\%$ humidity. A series of experiments were performed to assess the optimal parameters including printing contact time (1-5 s), various viscosity regulating buffers (0-5\% glycerol, 0-1\% PEG 400 and $0-1 \%$ tween 20), as well as the printing tip size $(30 \mu \mathrm{m}$ and $60 \mu \mathrm{m})$. We established the optimized printing parameters at $8 \times 8$ array with $250 \mu \mathrm{m}$ spacing and a contact time with the tip of $3 \mathrm{~s}$ with $5 \%$ glycerol. A single $1 \mathrm{~cm}^{2}$ substrate was printed with 4 such arrays (see figure 2C), demarked with hydrophobic pen such as to be able to study 4 samples simultaneously on the same substrate.

Assay development. The assay was developed as depicted in figure $3 \mathrm{~A}$. The protein $\mathrm{G}$ printed microarray with 64 individual micro-spots per array was incubated at RT for $2 \mathrm{~h}$. Each further step was thus performed ex-situ by static incubation of a biomolecule, followed by a dip and rinse step of PBS and milli-Q water for 3-4 min each, and drying with nitrogen. Each step was measured with the interferometric instrument to monitor the functionalization protocol and the subsequent detection. The optical image and read-out OPD values at each step are provided in figure 3B and 3C respectively. After protein $\mathrm{G}$ incubation, rinsing and measurement, the unspotted areas of the substrate were blocked by incubating with bovine serum albumin (BSA) at $0.5 \%(\mathrm{w} / \mathrm{v})$ in PBS for $15 \min \left(100 \mu \mathrm{L}\right.$ for $1 \mathrm{~cm}^{2}$ substrate). Specific antibody for E. coli $(500 \mu \mathrm{g} / \mathrm{mL}$ in PBS, unless otherwise mentioned) was then added (100 $\mu \mathrm{L}$ incubated onto a $1 \mathrm{~cm}^{2}$ substrate for $1 \mathrm{~h}$ at room temperature). Three different polyclonal antibodies were evaluated: ab31499 (from Abcam, Cambridge, UK) and rPAB80 and mPAB1:13.5 (provided by Diesse Diagnostica Senese, Italy). After rinsing and drying, the asbiofunctionalized array was ready for bacteria detection. E. coli dispersions were prepared according to the instructions from ielab for bacterial pellets BAControl-5 and BACuanti by dissolving a bacterial pellet in milli-Q water. The concentration of bacteria was confirmed by counting the cells in one pellet (i.e. $3.4 \times 10^{6} \mathrm{cfu} / \mathrm{mL}$ calculated $v s 5.3 \times 10^{6} \mathrm{cfu} / \mathrm{mL}$ indicated by the supplier). Further preparations included centrifugation and resuspension in the buffer for discarding the growth media, multiple such aliquots combined to minimize batch variation. Then serial dilutions were carried out to achieve the desired concentrations. E. coli dispersions were frozen in small aliquots providing two stock solutions of initial concentrations of $4.6 \times 10^{3}$ cells $/ \mathrm{mL}$ and $5.6 \times 10^{6}$ cells $/ \mathrm{mL}$. Two concentrations (medium and high) was used to avoid excessive dilutions. Fresh bacteria solutions were prepared right before measurements, by thawing aliquots $(500 \mu \mathrm{L})$ and centrifuging them at $7500 \mathrm{rpm}$ at $4{ }^{\circ} \mathrm{C}$ for $10 \mathrm{~min}$. After discarding the supernatant, the pellet was re-dispersed using PBS (500 $\mu \mathrm{L})$. Further dilutions 
were prepared (both in PBS or plasma) to obtain specific concentrations and generate the calibration curves in standard buffer or plasma respectively. Plasma bacteria solutions were diluted 1:3 (v/v) with PBS or PBST (PBS containing 0.1\% w/v tween 20). Each $8 \times 8$ array in the substrate was incubated with a $10 \mu \mathrm{L}$ E. coli sample (either in buffer or plasma) for 30 min at RT and measured after rinsing and drying.

Hospital samples. The samples used in the current validation analysis had already been collected to constitute the Sepsis Bank, a large collection of samples handled by the Biobank Unit at the Vall d'Hebron Barcelona Campus (Vall d'Hebron University Hospital / Vall d'Hebron Research Institute (VHIR). Informed consent was obtained from all blood donors (healthy donors, SIRS patients as well as sepsis patients) and the protocol had previously been approved by the Clinical Research Ethics Committee of the Hospital with reference number PR(AG)11/2006. The Sepsis Bank was set up in the context of an H2020 research project; accordingly, all data was protected in accordance with the EU Data Protection Directive 95/46/EC “on the protection of individuals with regard to the processing of personal data and on the free movement of such data”. Specifically, all samples and related clinical data were anonymized, and no personally identifiable data has ever been included in any dataset transmitted outside of VHIR. Patient samples (aliquots of blood, plasma and serum) were recovered from the Sepsis Bank. We specifically used EDTA plasma aliquots that were collected from patients for our present study.

Clinical validation. Patient plasma samples $(200 \mu \mathrm{L})$ were diluted in PBST $(600 \mu \mathrm{L})$ and $10 \mu \mathrm{L}$ of it (containing $2.5 \mu \mathrm{L}$ of undiluted patient sample) were incubated for 30 min onto a $8 \times 8$ antibodyfunctionalized microarray. The $\mathrm{OPD}_{\text {total }}$ signal obtained was correlated with the calibration curve generated in $25 \%$ plasma (figure 5C) and then further multiplied 4 times to obtain the original bacteria concentration in the undiluted patient plasma (which have been depicted in figure 6).

\section{Acknowledgements}

We acknowledge the financial support from European Union's Horizon 2020 Research and Innovation program (RAIS Project, grant agreement 644956). We thank Diesse diagnostica senese SPA for providing two E. coli antibodies. The ICN2 is funded by the CERCA programme / Generalitat de Catalunya. The ICN2 is supported by the Severo Ochoa programme of the Spanish Ministry of Economy, Industry and Competitiveness (MINECO, grant no. SEV-2013-0295). ICN2 thanks FEDER funds from the European Regional Development Funds program (Project FICTS-1420-27 selected by 
MINECO). RT and VP acknowledge financial support from the Spanish Ministry of Economy and Competitiveness through the Severo Ochoa Programme for Centres of Excellence in R\&D (SEV-20150522) and OPTO-SCREEN (TEC2016-75080-R), from Fundacio Privada Cellex, from Generalitat de Catalunya through the CERCA program and AGAUR 2017 SGR 1634. RT acknowledges support from the International $\mathrm{PhD}$ fellowship program 'la Caixa' Severo Ochoa at ICFO.

\section{Author Contributions}

P.D designed, performed the experiments and data analysis and wrote the manuscript. N.FF and O.CL supported part of the experiments. M.C.E designed the experiments and provided technical guidance. L.M.L provided technical guidance and contributed to the manuscript. R.T and V.P designed the lensfree interferometric microscope. F.Y, H.A and A.B. provided the optimized nanoplasmonic substrates. A.F, J.C.RR and J.J.GL collected blood plasma samples from patients, analysed them at their microbiological facility and clinically categorized the patients. All authors discussed and commented on the manuscript.

\section{Additional information}

Supplementary information is available online and includes plots related to optimization of printing conditions (printing buffer, spot size and antibody concentration), reproducibility of the assay protocol and selection of the specific and sensitive antibody for the assay. The table comprises the information of the hospital patient samples.

\section{Competing financial interests}

The authors declare no competing interests.

\section{References}

(1) Jones, K. E.; Patel, N. G.; Levy, M. A.; Storeygard, A.; Balk, D.; Gittleman, J. L.; Daszak, P. Global Trends in Emerging Infectious Diseases. Nature 2008, 451 (7181), 990-993.

(2) Gaynes, R.; Edwards, J. R.; System, N. N. I. S. Overview of Nosocomial Infections Caused by 
Gram-Negative Bacilli. Clin. Infect. Dis. 2005, 41 (6), 848-854.

(3) Cohen, J. The Immunopathogenesis of Sepsis. Nature 2002, 420 (6917), 885-891.

(4) Dellinger, R. P.; Levy, M. M.; Carlet, J. M.; Bion, J.; Parker, M. M.; Jaeschke, R.; Reinhart, K.; Angus, D. C.; Brun-Buisson, C.; Beale, R.; et al. Surviving Sepsis Campaign: International Guidelines for Management of Severe Sepsis and Septic Shock: 2008. Intensive Care Med. 2008, 34 (1), 17-60.

(5) Dombrovskiy, Viktor Y., MPH; Martin, Andrew A.; Sunderram, Jagadeeshan; Paz, H. L. Rapid Increase in Hospitalization and Mortality Rates for Severe Sepsis in the United States: A Trend Analysis from 1993 to 2003. Crit. Care Med. 2007, 35 (5), 1244-1250.

(6) Rittirsch, D.; Flierl, M. A.; Ward, P. A. Harmful Molecular Mechanisms in Sepsis. Nat. Rev. Immunol. 2008, 8 (10), 776-787.

(7) Yébenes, J. C.; Ruiz-Rodriguez, J. C.; Ferrer, R.; Clèries, M.; Bosch, A.; Lorencio, C.; Rodriguez, A.; Nuvials, X.; Martin-Loeches, I.; Artigas, A.; et al. Epidemiology of Sepsis in Catalonia: Analysis of Incidence and Outcomes in a European Setting. Ann. Intensive Care 2017, 7 (1), 1-10.

(8) Ford, H.; Systems, H.; Re-, C. W. Early Goal-Directed Therapy in the Treatment of Severe Sepsis and Septic Shock. N. Engl. J. Med. 2001, 345 (19), 1368-1377.

(9) Kumar, A.; Roberts, D.; Wood, K. E.; Light, B.; Parrillo, J. E.; Sharma, S.; Suppes, R.; Feinstein, D.; Zanotti, S.; Taiberg, L.; et al. Duration of Hypotension before Initiation of Effective Antimicrobial Therapy Is the Critical Determinant of Survival in Human Septic Shock. Crit. Care Med. 2006, 34 (6), 1589-1596.

(10) Ferrer, R.; Martin-Loeches, I.; Phillips, G.; Osborn, T. M.; Townsend, S.; Dellinger, R. P.; Artigas, A.; Schorr, C.; Levy, M. M. Empiric Antibiotic Treatment Reduces Mortality in Severe Sepsis and Septic Shock from the First Hour: Results from a Guideline-Based Performance Improvement Program. Crit. Care Med. 2014, 42 (8), 1749-1755.

(11) Lee, A.; Mirrett, S.; Reller, L. B.; Weinstein, M. P. Detection of Bloodstream Infections in Adults: How Many Blood Cultures Are Needed? J. Clin. Microbiol. 2007, 45 (11), 3546-3548.

(12) Lazcka, O.; Campo, F. J. Del; Muñoz, F. X. Pathogen Detection: A Perspective of Traditional Methods and Biosensors. Biosens. Bioelectron. 2007, 22 (7), 1205-1217. 
(13) Opota, O.; Croxatto, A.; Prod'hom, G.; Greub, G. Blood Culture-Based Diagnosis of Bacteraemia: State of the Art. Clin. Microbiol. Infect. 2015, 21 (4), 313-322.

(14) Sinha, M.; Jupe, J.; Mack, H.; Coleman, T. P.; Lawrence, S. M.; Fraley, I. Emerging Technologies for Molecular Diagnosis of Sepsis. Clin Microbiol Rev 2018, 31 (2), 1-26.

(15) Vincent, P. J.; Hospital, E.; Opal, P. S. M.; Division, I. D.; Hospital, M.; Marshall, P. J. C.; Michael, S.; Tracey, P. K. J. Sepsis Definitions: Time for Change. Lancet 2015, 381 (9868), 774-775.

(16) Gomez-Cruz, J.; Nair, S.; Manjarrez-Hernandez, A.; Gavilanes-Parra, S.; Ascanio, G.; Escobedo, C. Cost-Effective Flow-through Nanohole Array-Based Biosensing Platform for the Label-Free Detection of Uropathogenic E. Coli in Real Time. Biosens. Bioelectron. 2018, 106 (November 2017), 105-110.

(17) Maldonado, J.; González-Guerrero, A. B.; Domínguez, C.; Lechuga, L. M. Label-Free Bimodal Waveguide Immunosensor for Rapid Diagnosis of Bacterial Infections in Cirrhotic Patients. Biosens. Bioelectron. 2016, 85, 310-316.

(18) Liu, Y.; Zhou, H.; Hu, Z.; Yu, G.; Yang, D.; Zhao, J. Label and Label-Free Based SurfaceEnhanced Raman Scattering for Pathogen Bacteria Detection: A Review. Biosens. Bioelectron. 2017, 94 (December 2016), 131-140.

(19) Müller, V.; Sousa, J. M.; Ceylan Koydemir, H.; Veli, M.; Tseng, D.; Cerqueira, L.; Ozcan, A.; Azevedo, N. F.; Westerlund, F. Identification of Pathogenic Bacteria in Complex Samples Using a Smartphone Based Fluorescence Microscope. RSC Adv. 2018, 8 (64), 36493-36502.

(20) Zhu, H.; Sikora, U.; Ozcan, A. Quantum Dot Enabled Detection of Escherichia Coli Using a Cell-Phone. Analyst 2012, 137 (11), 2541-2544.

(21) Korhonen, T. K.; Valtonen, M. V; Parkkinen, J.; Vaisanen-Rhen, V.; Finne, J.; Orskov, F.; Orskov, I.; Svenson, S. B.; Makela, P. H. Serotypes, Hemolysin Production, and Receptor Recognition of Escherichia Coli Strains Associated with Neonatal Sepsis and Meningitis. Infect. Immun. 1985, 48 (2), 486-491.

(22) Ferens, W. A.; Hovde, C. J. Escherichia Coli O157:H7: Animal Reservoir and Sources of Human Infection. Foodborne Pathog. Dis. 2011, 8 (4), 465-487.

(23) Terborg, R. A.; Pello, J.; Mannelli, I.; Torres, J. P.; Pruneri, V. Ultrasensitive Interferometric 
On-Chip Microscopy of Transparent Objects. Sci. Adv. 2016, 2 (6), 1-7.

(24) Yesilkoy, F.; Terborg, R. A.; Pello, J.; Belushkin, A. A.; Jahani, Y.; Pruneri, V.; Altug, H. Phase-Sensitive Plasmonic Biosensor Using a Portable and Large Field-of-View Interferometric Microarray Imager. Light Sci. Appl. 2018, 7 (2), 17152.

(25) Ginger, D. S.; Zhang, H.; Mirkin, C. A. The Evolution of Dip-Pen Nanolithography. Angew. Chemie - Int. Ed. 2004, 43 (1), 30-45.

(26) Lee, K. B.; Lim, J. H.; Mirkin, C. A. Protein Nanostructures Formed via Direct-Write Dip-Pen Nanolithography. J. Am. Chem. Soc. 2003, 125 (19), 5588-5589.

(27) M, B. O. A.; Brodin, T.; Reis, K. Protein G : A Powerful Tool for Binding and Detection of Monoclonal and Polyclonal Antibodies. J. Immunol. 2018, 135, 2589-2592.

(28) Ahmed, A.; Rushworth, J. V.; Hirst, N. A.; Millner, P. A. Biosensors for Whole-Cell Bacterial Detection. Clin. Microbiol. Rev. 2014, 27 (3), 631-646.

(29) Yoo, S. M.; Lee, S. Y. Optical Biosensors for the Detection of Pathogenic Microorganisms. Trends Biotechnol. 2016, 34 (1), 7-25.

(30) Ivnitski, D.; Abdel-Hamid, I.; Atanasov, P.; Wilkins, E. Biosensors for Detection of Pathogenic Bacteria. Biosens. Bioelectron. 1999, 14 (7), 599-624.

(31) Chung, H. J.; Castro, C. M.; Im, H.; Lee, H.; Weissleder, R. A Magneto-DNA Nanoparticle System for Rapid Detection and Phenotyping of Bacteria. Nat. Nanotechnol. 2013, 8 (5), 369375.

(32) Yilmaz, E.; Majidi, D.; Ozgur, E.; Denizli, A. Whole Cell Imprinting Based Escherichia Coli Sensors: A Study for SPR and QCM. Sensors Actuators, B Chem. 2015, 209, 714-721.

(33) Bacconi, A.; Richmond, G. S.; Baroldi, M. A.; Laffler, T. G.; Blyn, L. B.; Carolan, H. E.; Frinder, M. R.; Toleno, D. M.; Metzgar, D.; Gutierrez, J. R.; et al. Improved Sensitivity for Molecular Detection of Bacterial and Candida Infections in Blood. J. Clin. Microbiol. 2014, 52 (9), 3164-3174.

(34) Metzgar, D.; Frinder, M. W.; Rothman, R. E.; Peterson, S.; Carroll, K. C.; Zhang, S. X.; Avornu, G. D.; Rounds, M. A.; Carolan, H. E.; Toleno, D. M.; et al. The IRIDICA BAC BSI Assay: Rapid, Sensitive and Culture-Independent Identification of Bacteria and Candida in Blood. 
PLoS One 2016, 11 (7), 1-16.

(35) Peters, R. P. H.; van Agtmael, M. A.; Gierveld, S.; Danner, S. A.; Groeneveld, A. B. J.; Vandenbroucke-Grauls, C. M. J. E.; Savelkoul, P. H. M. Quantitative Detection of Staphylococcus Aureus and Enterococcus Faecalis DNA in Blood To Diagnose Bacteremia in Patients in the Intensive Care Unit. J. Clin. Microbiol. 2007, 45 (11), 3641-3646. 


\section{TOC (Graphical abstract)}

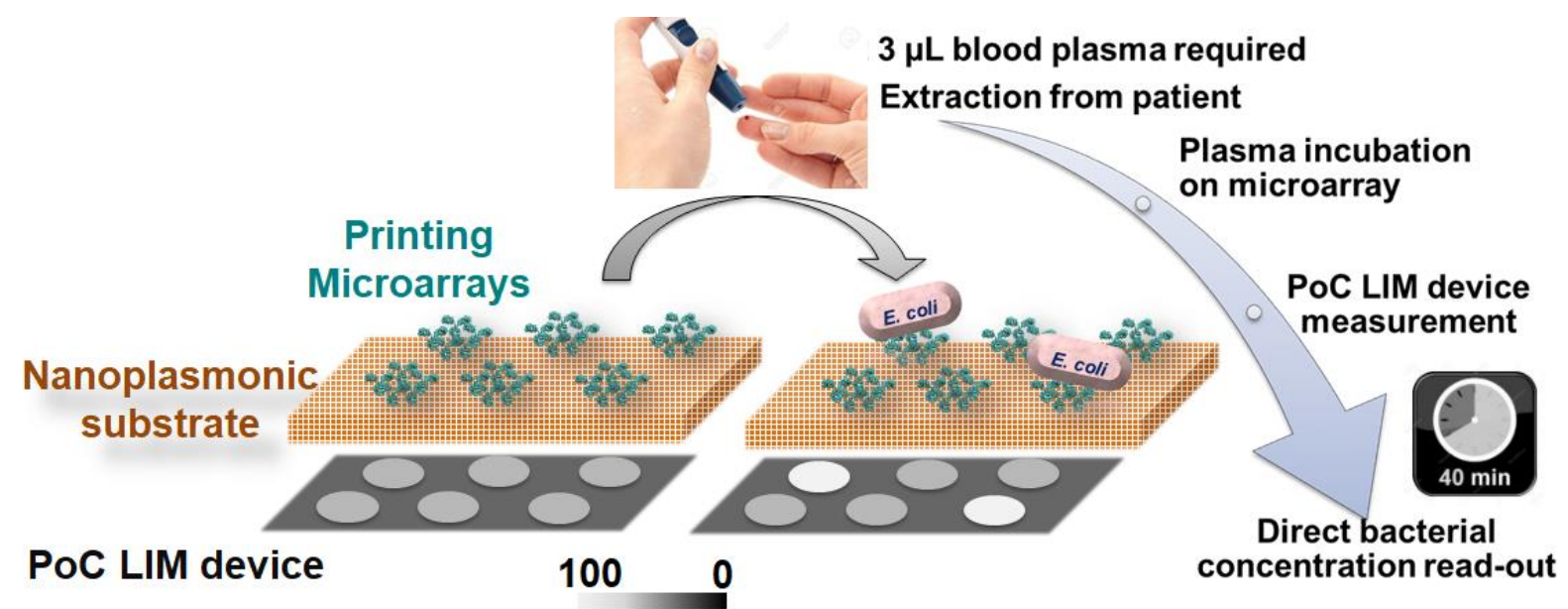

